\title{
A Combination Treatment of Antioxidants, Bone Graft and Platelet-rich Fibrin Increases the Number of Osteoblasts in the Post-tooth Extraction Socket of Wistar Rats
}

\author{
Hengky Marlie, Indira Apriantika, Muhammad Rubianto and Ernie Maduratna \\ Department of Periodontology, Faculty of Dental Medicine, Universitas Airlangga, Surabaya, Indonesia
}

\begin{abstract}
Background. Worldwide, periodontitis has the highest prevalence among the oral diseases. New medications are needed to repair the damage brought by periodontitis. Previous studies have been done to investigate agents such as antioxidants and platelet-rich fibrin (PRF) in oxidative stress. These studies focused on the role of antioxidants in periodontitis and other related systemic conditions. The aim of this study was to determine if antioxidants in combination with bone graft and PRF can increase the number of osteoblasts for new bone growth.
\end{abstract}

Methods. Twenty-seven (27) Wistar rats were randomly allocated into 3 groups. In the first group, the postextraction tooth socket was filled with blood (control). In the second group, the socket was filled with antioxidants and bone graft. In the third group, the socket was filled with antioxidants, bone graft, and PRF. On day 14, the rats were euthanized and the tissue fixations were stained with hematoxylin-eosin (HE) for histologic examination of the osteoblasts. One-way ANOVA was used as statistical test.

Results. Statistical test showed that there were significant differences in the number of osteoblasts in all the 3 groups $(p=0.000)$.

Conclusion. The number of osteoblasts was significantly increased in the post-extraction tooth socket treated with a combination of antioxidants, bone graft, and PRF.

Key Words: periodontal disease, antioxidant. osteoblasts, osteoclasts, platelet-rich fibrin

\section{INTRODUCTION}

Paper presented at the Joint Scientific Meeting in Special Care Dentistry, July 5, 2019, Amerta Room, 4th Floor, main campus of Universitas Airlangga, Surabaya, Indonesia.

Corresponding author: Ernie Maduratna

Department of Periodontology

Faculty of Dental Medicine

Universitas Airlangga

JI. Mayjen. Prof. Dr. Moestopo No. 47 Surabaya 60132 - Indonesia

Email: erniemaduratna@gmail.com
Periodontitis is one of the most pressing oral health problems in the world. The prevalence is high, affecting around $10-15 \%$ of the world population; untreated periodontitis can cause tooth loss. ${ }^{1}$ Periodontitis is caused by an imbalance between the bacterial plaque and the immunity status of the host. ${ }^{2}$ It is an inflammatory disease that can lead to destruction of the alveolar bone, cementum, and periodontal ligament. It occurs as a result of microbial invasion on tooth surface. ${ }^{3}$

The pathogenic bacteria can release lipopolysaccharide toxin which can damage the periodontal tissue, especially in the alveolar bone. The stimulation induces the activation of osteoclasts and decrease of osteoblasts; this will lead to the destruction of inorganic minerals from the alveolar bone, thus, triggering the resorption of alveolar bone. ${ }^{4}$

Pathogenic bacteria also stimulate the response of immune cells from the host to produce a variety 
of interleukin (IL) and tumor necrosis factor-alpha $(\mathrm{TNF}-\alpha){ }^{4}$ These pro-inflammatory cytokines will attract polymorphonuclear neutrophils (PMN) to the infection site, leading to the production of free radicals, reactive oxygen species (ROS). ROS stimulates increase in osteoclasts which lead to bone resorption. ${ }^{5}$

Alveolar bone is formed by osteoblasts. It is absorbed by active osteoclasts. Osteoblasts are not only found on the outer surface of the bone but also in the cavities of the bone. A small number of the osteoblastic process occurs in all of the bone living tissues. New bones are formed constantly. ${ }^{6}$

The osteoblast is responsible for bone formation. It is derived from the stromal cell of the bone marrow. Osteoblast produces collagen type 1 and alkaline phosphatase during the developmental and maturation process. Bone mineralization occurs in the presence of hydroxyapatite (HA) crystals in an organic bone matrix consisting of type I collagen and several other proteins. ${ }^{7}$

In normal alveolar bone, formation and maintenance processes are regulated by the balance of osteoblasts and osteoclasts; an imbalance between osteoblasts and osteoclasts can lead to a pathogenetic process which is the main cause of bone disease. ${ }^{6}$ During the said process, an imbalance between bone resorption and bone formation will increase resorption. Differentiation and activity of osteoclasts are stimulated by receptor activity nuclear factor kappa-B ligand (RANKL) on the osteoblasts and receptor activity nuclear factor kappa-B (RANK) on osteoclast precursors with their macrophage colony stimulating factor (M-CSF). Binding of RANK and RANKL induces signal transduction through the nuclear factor kappa B (NF-kB); this phase triggers differentiation of precursor cells into osteoclast preosteoclasts. Some inflammatory cytokines including IL$1, \mathrm{TNF}-\alpha$, and IL- 6 can increase the production of RANK which indicates that the inflammation can cause bone damage. In addition, TNF- $\alpha$ may increase osteoclast activity by inducing RANKL signaling. ${ }^{8}$

The treatment goal is not only to stop periodontal disease but also to regenerate the damaged site of periodontal tissue. $^{9}$ Regeneration occurs because the growth and differentiation of cells and cellular substances form the new periodontal tissue. It derives from the same type of tissue or from their precursors. Periodontal regeneration includes repair of alveolar bone, cementum, and periodontal ligament. ${ }^{10}$ There are many materials and techniques that have been used to stimulate the growth and differentiation of cells such as bone graft, guided tissue regeneration (GTR), growth factor or other materials. ${ }^{11}$

Bone graft is mostly used to repair bone damage in periodontal disease. It can help the process of bone regeneration through two mechanisms, which are osteoinduction and osteoconduction. ${ }^{11}$

Furthermore, there are three processes to bone healing including reactive phase, reparative phase, and remodeling phase. The reactive and reparative phases take around 6-8 weeks. The remodeling phase lasts up to several months or a year. ${ }^{12}$ The healing process of bone can be clearly visible on radiographic examination after 3 months of bone graft procedure. In the sequel, the process of mineralization, bone density will increase significantly in the six months after bone graft implantation. ${ }^{13}$ Moreover, the alveolar bone defect healing process is a structured process and it is influenced by several things such as bacterial contamination, the characteristics of periodontal breakdown, operating procedures, and general factors that potentially deter healing such as age, genetics, smoking, and diabetes mellitus. ${ }^{14}$

Previous studies indicate that platelet-rich fibrin (PRF) has had an important role in stimulating periodontal regeneration. $\mathrm{PRF}$ is the product of autologous platelets and is rich in growth factors and cytokines that can increase the potential for hard and soft tissue healing. Two growth factors that have a prominent effect on bone healing are plateletderived growth factor (PDGF) and TGF- $\beta$. Both are the most important mediators to stimulate osteogenesis in bone defects. The addition of PRF on bone graft is known to accelerate growth and bone healing. ${ }^{15}$

Antioxidants and PRF are known to reduce the damaging effects of oxidative stress on cells. Antioxidants can neutralize various types of oxidative stress. Previous studies have examined the role of antioxidants such as enzymes catalase (CAT), glutathione peroxidase (GPx), and superoxide dismutase (SOD) as marker of defense mechanisms against bone resorption. ${ }^{16}$

A few researches focused on the role of antioxidants to help reduce periodontal destruction because of oxidative stress. There have been limited studies concerning the mechanism of action of antioxidants in the regenerative therapy for periodontal disease. Research is needed to see if antioxidants in combination with bone graft and PRF can lend support to the tissue-engineering scaffold provided by $\mathrm{PRF}$ for regenerative therapy involving increase in the number of osteoblasts. Thus, this research aimed to determine the effect of the combination of antioxidants, bone graft, and PRF in alveolar bone and in the healing process of periodontal disease.

\section{MATERIALS AND METHODS}

\section{Animal Management Experiment}

The research was an experimental laboratory study with posttest-only control design. There were 27 Wistar rats which were randomly allocated to three different treatment groups. The study protocol was approved by the Ethical Committee of Faculty of Dental Medicine Universitas Airlangga (No. 198/KKEPK.FKG/XI/2015).

The incisive mandibular teeth of the study animals were extracted. In the first group of animals, the socket was left untreated. In the second group, the socket was filled with antioxidants (apple extract, Indonesia) and bone graft (Bio Hydrox, Dr. Soetomo Tissue Bank, Indonesia). In the 
third group, the socket was filled antioxidants, bone graft, and PRF. After a surgical procedure 14 days later, expression of osteoblasts in the socket was seen.

\section{Preparation of $\mathrm{PRF}^{17}$}

PRF was prepared from $5 \mathrm{~mL}$ of each rat's blood. Blood was collected and put into sterile tubes without anticoagulant; the tubes were centrifuged for 10 minutes at $2500 \mathrm{rpm}$. When centrifugation was finished, the resultant products were divided into 3 parts, the bottom was red blood, the middle was the PRF, and the upper part was acellular plasma.

\section{Application of Antioxidants, Bone graft, and PRF ${ }^{12}$}

The rats were administered intramuscularly with ketamine $2 \%$ at $15-25 \mathrm{mg} / \mathrm{kg}$ body weight, as general anaesthesia. Before extraction, the tooth was injected with local anaesthetic lidocaine $1 \mathrm{~mL}$ to strengthen the anaesthesia effect. A mandibular incisor tooth was extracted. The socket was irrigated with saline. For the first group, the socket was filled with blood (control). For the second group, the socket was filled with antioxidants and bone graft. For the third group, the socket was filled antioxidants, bone graft, and PRF. The wound was then sutured.

\section{Sample}

On the $14^{\text {th }}$ day, the experimental animals were euthanized by injection of ketamine $50 \mathrm{mg} / \mathrm{kg}$ body weight. When breathing had stopped, each jaw was taken using a circular saw. The jaw was then stored in a buffer of $70 \%$ formalin solution in order to prevent tissue changes and to forestall decomposition and tissue hardening, and to increase the refractive index of the various network components and the affinity network against paint materials. After the first 48 hours the fixative was replaced, and a new and smaller cut tissue fixation was prepared so the fixative can penetrate evenly into the network. In the second stage, the tissue fixation was left in the solution for 48 hours. The tissue fixation was then rinsed with running water for 6-9 hours and was put in a decalcification solution of $5 \% \mathrm{HNO} 3$ for 1 hour. The steps that followed included:

1. Dehydration (extraction of water from the network)

2. Clearing (purification)

3. Impregnation at a temperature of $56^{\circ}$ Celsius in paraffin bedding

4. Cutting with a microtome, with a thickness of 4 microns. Then HE staining (hematoxylin-eosin)

\section{Assessment of Results}

The number of osteoblasts was counted on the samples with Hematoxylin-Eosin (HE) stain. The results were calculated using a microscope with a magnification of 1000x. The results were then recorded.

\section{Data analysis}

The study used one-way ANOVA to determine the differences between the groups. If there were significant differences, Tukey HSD test was then used.

Observation of the Hematoxylin-Eosin (HE) results for histology examination done. Each group was observed with a microscope OLYMPUS BX series 41. This microscope was equipped with a DP-70 digital camera and used software OLYSIA. The intensity of the color in osteoblasts area can be considered as a quantitative value.

Figures 1-3 show the combination of antioxidants, bone graft, and PRF showed increase in the the number of osteoblasts compared to the control group and the antioxidants and bone graft group. The results are also shown in Table 1 and Figure 4.

Figure 4 shows the number of osteoblasts was highest in antioxidants plus bone graft plus PRF group, with the average 15.00 .

\section{Data analysis}

The results of the study were tested for normality and homogeneity of the research data; the KolmogorovSmirnov test was used for normality and the Levene test for homogeneity. The Kolmogorov-Smirnov test results indicated that the probability of each treatment group was greater than $0.05(p>0.05)$, which means that all the data were pulled from a symmetric or normal distribution. Results of the Levene test on osteoblasts was $\mathrm{p}=0.169$, which means the data were homogeneous ( $p>0.05)$. Therefore, the data obtained in our study have been shown to be normally distributed and homogeneous. The one-way ANOVA test was then used to determine differences in the number of osteoblasts between the groups (Table 2). The results showed that antioxidants plus bone graft plus PRF combination can provide support structures or scaffolding for regenerative therapy that can result in the increase of the number of osteoblasts.

Table 2 shows the results of parametric test and oneway ANOVA $\mathrm{p}$-value $=0.000 \quad(\mathrm{p}<0.05) \quad$ which means

Table 1. Mean of Osteoblasts

\begin{tabular}{cccc} 
No. & Treatment group & N & Mean \\
\hline 1 & Control & 9 & 3.56 \\
2 & Antioxidants and Bone graft & 9 & 9.33 \\
3 & Antioxidants, Bone graft PRF & 9 & 15.00 \\
\hline
\end{tabular}

Table 2. One-way ANOVA Test Results on the Number of Osteoblasts in Control Group, Antioxidants and Bone graft Group, and Combination Antioxidants, Bone graft, and PRF Group

\begin{tabular}{lrrrrr} 
& Sum of square & df & $\begin{array}{r}\text { Mean } \\
\text { Square }\end{array}$ & F & Sig \\
\hline Between groups & 589,407 & 2 & 29,704 & 103,674 &, 000 \\
Within groups & 68,222 & 24 & 2,843 & & \\
$\quad$ Total & 657,630 & 26 & & & \\
\hline
\end{tabular}




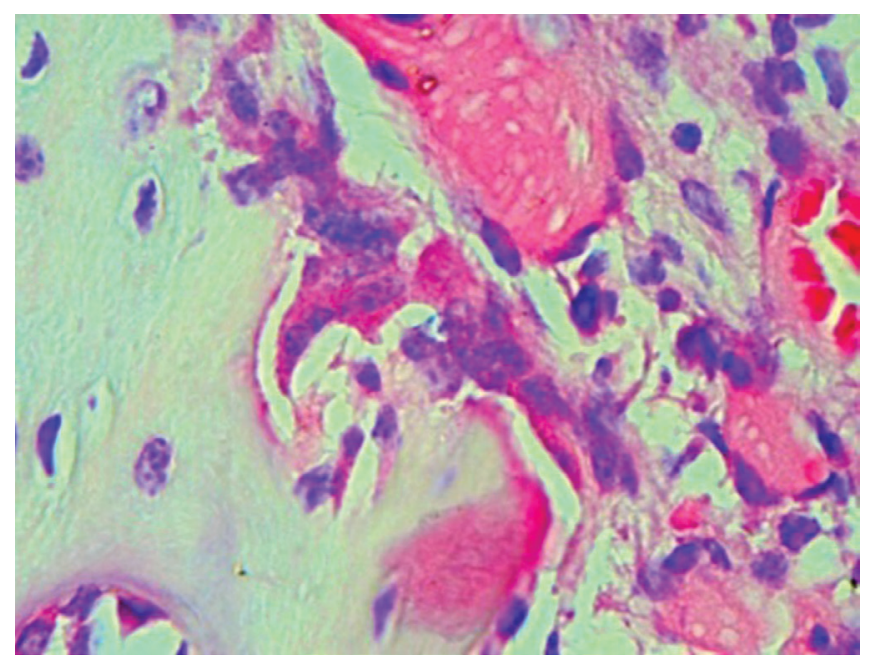

Figure 1. The osteoblasts in the control group (staining $\mathrm{H} \& \mathrm{E}$ and magnification $\times 1000$ ).

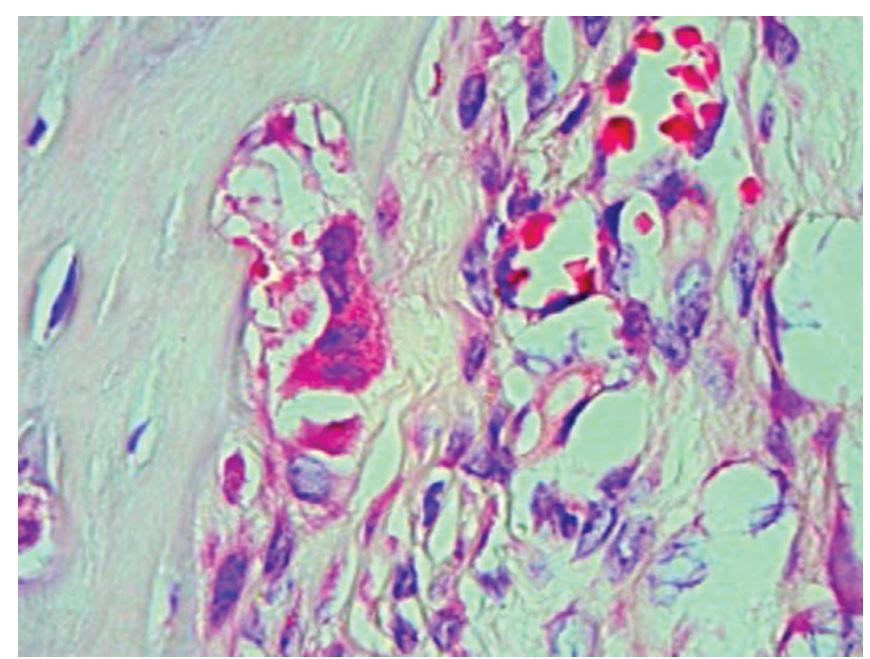

Figure 2. The osteoblasts in the antioxidants + bone graft group (staining $\mathrm{H} \& \mathrm{E}$ and magnification $\times 1000$ ).

that there were significant differences in the number of osteoblasts between the treatment groups. Furthermore, the Post Hoc test was conducted to know which groups have a significant difference.

The results of the Post Hoc test between treatment groups was 0.000 . The probability was less than $0.05(\mathrm{p}<0.05)$ means that there were significant differences in the mean number of osteoblasts between the groups.

\section{DISCUSSION}

Bone is composed of 4 types of bone cells. They are osteoprogenitor cells, osteoblasts, osteocytes, and osteoclasts. Osteoblasts synthesize organic matrix element; osteocytes, which have cavities (lacunae) in the matrix; and osteoclasts which are multinuclear giant cells involved in resorption and remodeling of bone tissue. Bone has the ability to

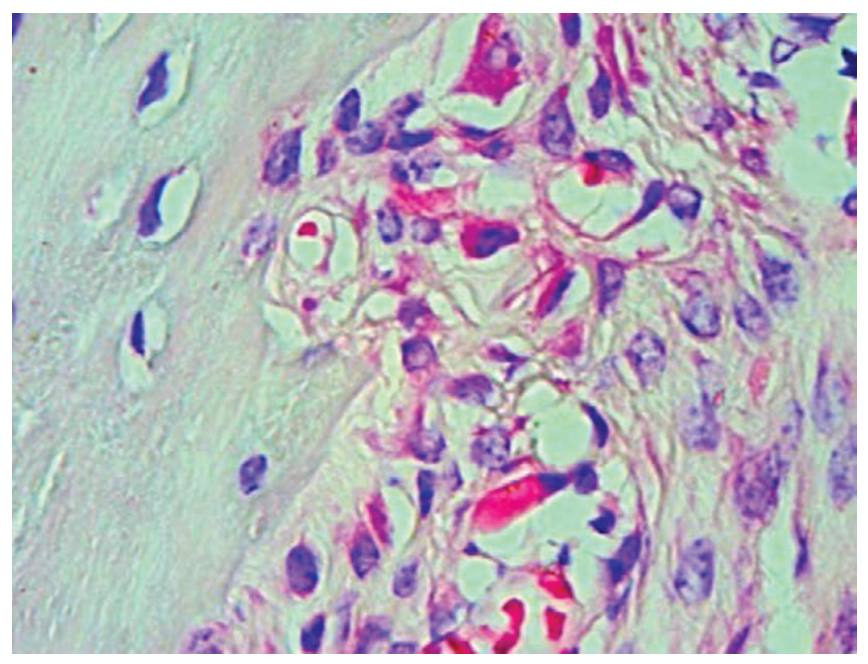

Figure 3. The osteoblasts in antioxidants + bone graft + PRF (staining H\&E and magnification x1000).

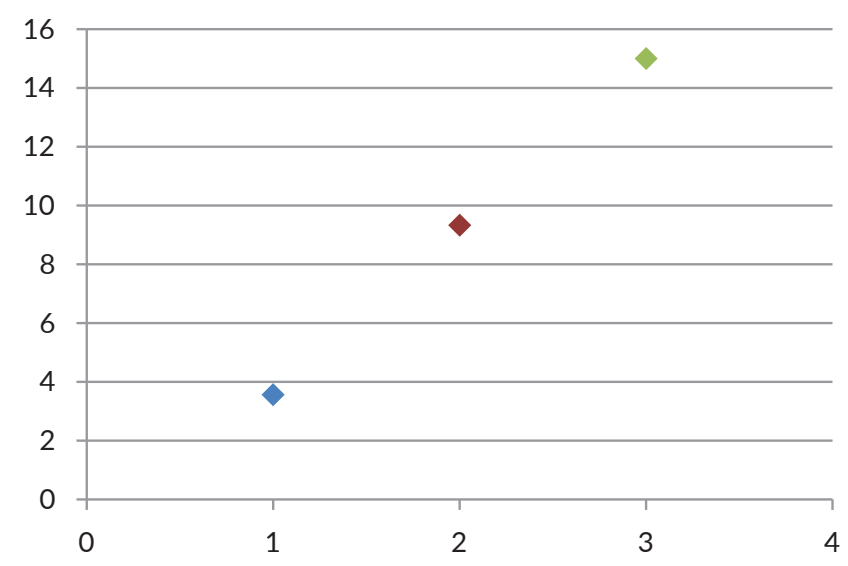

$\diamond$ Control $\diamond$ Antioxidants+bone graft $\diamond$ Antioxidants+bone graft+PRF

Figure 4. Number of Osteoblasts in Scatter Chart.

regenerate. Osteoblasts synthesize new bone formation because the osteoblast secretes osteocytes and modulates the occurrence of hydroxyapatite crystals. ${ }^{6}$

The process of periodontal tissue regeneration using a material that is bone graft showed that this could be useful. Bone graft can help the process of bone regeneration. Besides bone graft as scaffold, the bone healing process is also influenced by the growth factor. Growth factors involved in the formation of osteoblasts include, among others, IGF, PDGF, and TGF-B. All three are key mediators in the process of bone healing. Bone graft often used in periodontal flap surgical procedure that is expected to help prevent the growth of new bone through the process of osteogenesis, osteoinduction, and osteoconduction may not be enough. ${ }^{18}$

Previous study focused on antioxidants showed that antioxidants may help to reduce periodontal destruction due to oxidative stress. Therefore, the purpose of this 
study was to determine the increase in the number of osteoblasts in Wistar Rats post-extraction socket treated by the combination of antioxidant, bone graft, and PRF. The combination of these three materials resulted in the increase of the new bone growth.

In this study, an examination performed on day 14 showed that the highest number of osteoblasts was in the combination group of antioxidants, bone graft, and PRF, compared with the control group and the combination antioxidants and bone graft group. Bone formation begins at day 10 to day 12, wherein PRF growth factors such as PDGF and TGF will help regenerate bone. PDGF stimulates chemotaxis, mitogenesis, and replication of stem cells in the wound to form the bone matrix and angiogenesis, stimulating the production of fibronectin, cell adhesion molecule that plays a role in cell migration and proliferation and remodeling. TGF- $\beta 1$ and TGF- $\beta 2$ also have a role in bone and tissue regeneration. ${ }^{19}$

The addition of antioxidants provides structural support to reduce the amount of excessive ROS so that the inflammatory phase is short and the process is accelerated into the reparative phase. Giving antioxidants can provide stability to the healing process and may prevent the damaging effects of ROS. The high number of osteoblasts may prevent the production of osteoclasts in a manner that there will be osteoblasts producing osteoprotegerin (OPG) for RANKL to bind to RANK so it can not bind to the receptor RANK, so that the number of osteoclasts will decline. ${ }^{20}$

PRF is also known to accelerate the proliferation and differentiation of cells that play a role in periodontal regeneration. Platelet activation will release growth factors that play a role in all phases of healing. Growth factor secretion occur within 1 hour, followed by additional protein secretion within 5-10 days. ${ }^{21}$

The inflammatory phase is important because accompanied by an increase in ROS it can inhibit the healing process by causing damage to osteoblasts and fibroblasts. They will inhibit the formation of granulation tissue and reparative phase delays. Moreover, the inflammatory phase can be maintained over time and prevent entry into the reparative phase. This can occur when the inflammatory phase is reinitiated as when the damaged bone structure is not supported. In this case, the lack of support for the damaged bone can cause additional inflammatory phase. ${ }^{16}$

The body's defence system produces an endogenous antioxidant, but in inflammatory conditions, the immune system can not produce endogenous antioxidant in sufficient quantity. Therefore, there is a need for exogenous antioxidants to reduce the damaging effects of ROS. Antioxidants can promote osteogenic process and the synthesis of collagen transcript thus they can facilitate the express osteoblast activity and reduce the damaging effects of ROS. Antioxidants can increase the amount of OPG. It blocks osteoclast formation and bone resorption by inhibiting the bond between RANK and RANKL so osteoclastogenesis does not happen. ${ }^{8}$
Statistical test using one-way ANOVA has proved that there were significant differences in the number of osteoblasts between the groups. This research showed osteoblasts well respond to antioxidants for the healing process in the sockets of Wistar rats. The combination of antioxidants, bone graft, and PRF can be used for periodontal regeneration resulting from increase in the the number of osteoblasts compared with the combination antioxidants and bone graft, as well as the control group. Antioxidants combination bone graft and PRF can provide support structures for regenerative therapies.

\section{CONCLUSION}

The number of osteoblasts was increased in Wistar rats post-extraction socket treated by combination of antioxidant, bone graft, and PRF.

\section{Statement of Authorship}

All authors participated in data collection and analysis, and approved the final version submitted.

\section{Author Disclosure}

All authors declared no conflict of interest.

\section{Funding Source}

None.

\section{REFERENCES}

1. Rajendran D, Murugan S, Subbulakshmi P, Saravanan R. Antioxidants in Periodontal Diseases. Critical Review in Pharmaceutical Sciences. 2013; 2(2):7-12.

2. Trivedi S, Lal N. Antioxidant enzymes in periodontitis. J Oral Biol Craniofac Res. 2017 Jan-Apr; 7(1):54-57.

3. Kurgan S, Kantarci A. Molecular basis for immunohistochemical and inflammatory changes during progression of gingivitis to periodontitis. Periodontol 2000. 2018 Feb; 76(1):51-67.

4. Hienz SA, Paliwal S, Ivanovski S. Mechanisms of Bone Resorption in Periodontitis. J Immunol Res. 2015; 2015:615486.

5. Kanzaki H, Wada S, Narimiya T, Yamaguchi Y, Katsumata Y, Itohiya K, et al. Pathways that Regulate ROS Scavenging Enzymes, and Their Role in Defense Against Tissue Destruction in Periodontitis. Front Physiol. 2017 May; 8:351

6. Jiang N, Guo W, Chen M, Zheng Y, Zhou J, Kim SG, et al. Periodontal Ligament and Alveolar Bone in Health and Adaptation: Tooth Movement. Front Oral Biol. 2016; 18:1-8.

7. Chen X, Wang Z, Duan N, Zhu G, Schwarz EM, Xie C. Osteoblastosteoclast interactions. Connect Tissue Res. 2018 Mar; 59(2):99-107.

8. Hubert PA, Lee SG, Lee SK, Chun OK. Dietary Polyphenols, Berries, and Age-Related Bone Loss: A Review Based on Human, Animal, and Cell Studies. Antioxidants. 2014 Mar; 3(1):144-58.

9. Sivaram G, Nandhini Devi RS. Stem Cells in Periodontal Regeneration. IOSR J Dent Med Sci. 2014;13(9):31-40.

10. Newman MG, Takei HH, Klokkevold PR, Carranza F. Carranza's Clinical Periodontology. Saunders; 2014.

11. Wang W, Yeung KWK. Bone grafts and biomaterials substitutes for bone defect repair: A review. Bioact Mater. 2017 Jun; 2(4):224-47.

12. Setiawatie EM. Hydroxyapatite combined with hyaluronic acid metronidazole gel increased the quantity of osteoblasts in the alveolar bone wistar rat. Dental Journal (Majalah Kedokteran Gigi) 2015 Dec; 48(4):204-8. 
13. Wall A, Board T. Bone morphogenetic protein. In: Classic Papers in Orthopaedics. 2014.

14. Larsson L, Decker AM, Nibali L, Pilipchuk SP, Berglundh T, Giannobile WV. Regenerative Medicine for Periodontal and Periimplant Diseases. J Dent Res. 2016 Mar; 95(3):255-66.

15. Choukroun J, Diss A, Simonpieri A, Girard MO, Schoeffler C, Dohan $\mathrm{SL}$, et al. Platelet-rich fibrin (PRF): A second-generation platelet concentrate. Part IV: Clinical effects on tissue healing. Oral Surg Oral Med Oral Pathol Oral Radiol Endod. 2006 Mar; 101(3):56-60.

16. Kurutas EB. The importance of antioxidants which play the role in cellular response against oxidative/nitrosative stress: current state. Nutr J. 2016; 15(1):71.

17. Duan X, Lin Z, Lin X, Wang Z,Wu Y,Ji M, et al. Study of platelet-rich fibrin combined with rat periodontal ligament stem cells in periodontal tissue regeneration. J Cell Mol Med. 2018 Feb; 22(2):1047-55.
18. Farid SBH. Osteoinduction, osteoconduction, and osseointegration. In: Bioceramics: For Materials Science and Engineering. 2019.

19. Albanese A, Licata ME, Polizzi B, Campisi G. Platelet-rich plasma (PRP) in dental and oral surgery: From the wound healing to bone regeneration. Immun Ageing. 2013 Jun; 10(1):23.

20. Tomofuji T, Ekuni D, Mizutani S, Morita M. Oxidative Stress in Applied Basic Research and Clinical Practice. 2014. pp 279-305.

21. Fernandes G, Yang S. Application of platelet-rich plasma with stem cells in bone and periodontal tissue engineering. Bone Res. 2016; 4:16036. 